\title{
The Definition of Topological Manifolds
}

\author{
Marco Riccardi \\ Via del Pero 102 \\ 54038 Montignoso, Italy
}

\begin{abstract}
Summary. This article introduces the definition of $n$-locally Euclidean topological spaces and topological manifolds [13].
\end{abstract}

MML identifier: MFOLD_1, version: $\underline{7.11 .07 \quad 4.156 .1112}$

The papers [8], [1], [6], [15], [7], [18], [3], [4], [17], [2], [16], [9], [19], [20], [11], $[12],[10],[14]$, and [5] provide the terminology and notation for this paper.

\section{Preliminaries}

Let $x, y$ be sets. Observe that $\{\langle x, y\rangle\}$ is one-to-one.

In the sequel $n$ denotes a natural number.

One can prove the following two propositions:

(1) For every non empty topological space $T$ holds $T$ and $T\left\lceil\Omega_{T}\right.$ are homeomorphic.

(2) Let $X$ be a non empty subspace of $\mathcal{E}_{\mathrm{T}}^{n}$ and $f$ be a function from $X$ into $\mathbb{R}^{1}$. Suppose $f$ is continuous. Then there exists a function $g$ from $X$ into $\mathcal{E}_{\mathrm{T}}^{n}$ such that

(i) for every point $a$ of $X$ and for every point $b$ of $\mathcal{E}_{\mathrm{T}}^{n}$ and for every real number $r$ such that $a=b$ and $f(a)=r$ holds $g(b)=r \cdot b$, and

(ii) $g$ is continuous.

Let us consider $n$ and let $S$ be a subset of $\mathcal{E}_{\mathrm{T}}^{n}$. We say that $S$ is ball if and only if:

(Def. 1) There exists a point $p$ of $\mathcal{E}_{\mathrm{T}}^{n}$ and there exists a real number $r$ such that $S=\operatorname{Ball}(p, r)$. 
Let us consider $n$. Observe that there exists a subset of $\mathcal{E}_{\mathrm{T}}^{n}$ which is ball and every subset of $\mathcal{E}_{\mathrm{T}}^{n}$ which is ball is also open.

Let us consider $n$. One can verify that there exists a subset of $\mathcal{E}_{\mathrm{T}}^{n}$ which is non empty and ball.

In the sequel $p$ denotes a point of $\mathcal{E}_{\mathrm{T}}^{n}$ and $r$ denotes a real number.

The following proposition is true

(3) For every open subset $S$ of $\mathcal{E}_{\mathrm{T}}^{n}$ such that $p \in S$ there exists ball subset $B$ of $\mathcal{E}_{\mathrm{T}}^{n}$ such that $B \subseteq S$ and $p \in B$.

Let us consider $n, p, r$. The functor $\mathbb{B}_{r}(p)$ yields a subspace of $\mathcal{E}_{\mathrm{T}}^{n}$ and is defined as follows:

(Def. 2) $\quad \mathbb{B}_{r}(p)=\mathcal{E}_{\mathrm{T}}^{n} \uparrow \operatorname{Ball}(p, r)$.

Let us consider $n$. The functor $\mathbb{B}^{n}$ yields a subspace of $\mathcal{E}_{\mathrm{T}}^{n}$ and is defined as follows:

(Def. 3) $\quad \mathbb{B}^{n}=\mathbb{B}_{1}\left(0_{\mathcal{E}_{\mathrm{T}}^{n}}\right)$.

Let us consider $n$. One can verify that $\mathbb{B}^{n}$ is non empty. Let us consider $p$ and let $s$ be a positive real number. Observe that $\mathbb{B}_{s}(p)$ is non empty.

The following propositions are true:

(4) The carrier of $\mathbb{B}_{r}(p)=\operatorname{Ball}(p, r)$.

(5) If $n \neq 0$ and $p$ is a point of $\mathbb{B}^{n}$, then $|p|<1$.

(6) Let $f$ be a function from $\mathbb{B}^{n}$ into $\mathcal{E}_{\mathrm{T}}^{n}$. Suppose $n \neq 0$ and for every point $a$ of $\mathbb{B}^{n}$ and for every point $b$ of $\mathcal{E}_{\mathrm{T}}^{n}$ such that $a=b$ holds $f(a)=\frac{1}{1-|b| \cdot|b|} \cdot b$. Then $f$ is homeomorphism.

(7) Let $r$ be a positive real number and $f$ be a function from $\mathbb{B}^{n}$ into $\mathbb{B}_{r}(p)$. Suppose $n \neq 0$ and for every point $a$ of $\mathbb{B}^{n}$ and for every point $b$ of $\mathcal{E}_{\mathrm{T}}^{n}$ such that $a=b$ holds $f(a)=r \cdot b+p$. Then $f$ is homeomorphism.

(8) $\mathbb{B}^{n}$ and $\mathcal{E}_{\mathrm{T}}^{n}$ are homeomorphic.

In the sequel $q$ denotes a point of $\mathcal{E}_{\mathrm{T}}^{n}$.

We now state three propositions:

(9) For all positive real numbers $r, s$ holds $\mathbb{B}_{r}(p)$ and $\mathbb{B}_{s}(q)$ are homeomorphic.

(10) For every non empty ball subset $B$ of $\mathcal{E}_{\mathrm{T}}^{n}$ holds $B$ and $\Omega_{\mathcal{E}_{\mathrm{T}}^{n}}$ are homeomorphic.

(11) Let $M, N$ be non empty topological spaces, $p$ be a point of $M, U$ be a neighbourhood of $p$, and $B$ be an open subset of $N$. Suppose $U$ and $B$ are homeomorphic. Then there exists an open subset $V$ of $M$ and there exists an open subset $S$ of $N$ such that $V \subseteq U$ and $p \in V$ and $V$ and $S$ are homeomorphic. 


\section{MANIFOLD}

In the sequel $M$ is a non empty topological space.

Let us consider $n, M$. We say that $M$ is $n$-locally Euclidean if and only if the condition (Def. 4) is satisfied.

(Def. 4) Let $p$ be a point of $M$. Then there exists a neighbourhood $U$ of $p$ and there exists an open subset $S$ of $\mathcal{E}_{\mathrm{T}}^{n}$ such that $U$ and $S$ are homeomorphic.

Let us consider $n$. Observe that $\mathcal{E}_{\mathrm{T}}^{n}$ is $n$-locally Euclidean.

Let us consider $n$. Observe that there exists a non empty topological space which is $n$-locally Euclidean.

We now state two propositions:

(12) $M$ is $n$-locally Euclidean if and only if for every point $p$ of $M$ there exists a neighbourhood $U$ of $p$ and there exists ball subset $B$ of $\mathcal{E}_{\mathrm{T}}^{n}$ such that $U$ and $B$ are homeomorphic.

(13) $M$ is $n$-locally Euclidean if and only if for every point $p$ of $M$ there exists a neighbourhood $U$ of $p$ such that $U$ and $\Omega_{\mathcal{E}_{\mathrm{T}}^{n}}$ are homeomorphic.

Let us consider $n$. Observe that every non empty topological space which is $n$-locally Euclidean is also first-countable.

Let us note that every non empty topological space which is 0-locally Euclidean is also discrete and every non empty topological space which is discrete is also 0-locally Euclidean.

Let us consider $n$. One can verify that $\mathcal{E}_{\mathrm{T}}^{n}$ is second-countable.

Let us consider $n$. Note that there exists a non empty topological space which is second-countable, Hausdorff, and $n$-locally Euclidean.

Let us consider $n, M$. We say that $M$ is $n$-manifold if and only if:

(Def. 5) $\quad M$ is second-countable, Hausdorff, and $n$-locally Euclidean.

Let us consider $M$. We say that $M$ is manifold-like if and only if:

(Def. 6) There exists $n$ such that $M$ is $n$-manifold.

Let us consider $n$. Observe that there exists a non empty topological space which is $n$-manifold.

Let us consider $n$. One can check the following observations:

* every non empty topological space which is $n$-manifold is also secondcountable, Hausdorff, and $n$-locally Euclidean,

* every non empty topological space which is second-countable, Hausdorff, and $n$-locally Euclidean is also $n$-manifold, and

* every non empty topological space which is $n$-manifold is also manifoldlike.

Let us note that every non empty topological space which is second-countable and discrete is also 0-manifold. 
Let us consider $n$ and let $M$ be an $n$-manifold non empty topological space. One can verify that every non empty subspace of $M$ which is open is also $n$ manifold.

Let us note that there exists a non empty topological space which is manifoldlike.

A manifold is a manifold-like non empty topological space.

\section{REFERENCES}

[1] Grzegorz Bancerek. Cardinal numbers. Formalized Mathematics, 1(2):377-382, 1990.

[2] Grzegorz Bancerek. The ordinal numbers. Formalized Mathematics, 1(1):91-96, 1990.

[3] Czesław Byliński. Functions and their basic properties. Formalized Mathematics, 1(1):5565, 1990.

[4] Czesław Byliński. Functions from a set to a set. Formalized Mathematics, 1(1):153-164, 1990.

[5] Czesław Byliński. Some basic properties of sets. Formalized Mathematics, 1(1):47-53, 1990.

[6] Agata Darmochwał. Compact spaces. Formalized Mathematics, 1(2):383-386, 1990.

[7] Agata Darmochwał. The Euclidean space. Formalized Mathematics, 2(4):599-603, 1991.

[8] Adam Grabowski. Properties of the product of compact topological spaces. Formalized Mathematics, 8(1):55-59, 1999.

[9] Krzysztof Hryniewiecki. Basic properties of real numbers. Formalized Mathematics, 1(1):35-40, 1990.

[10] Zbigniew Karno. Separated and weakly separated subspaces of topological spaces. Formalized Mathematics, 2(5):665-674, 1991.

[11] Zbigniew Karno. The lattice of domains of an extremally disconnected space. Formalized Mathematics, 3(2):143-149, 1992.

[12] Artur Korniłowicz and Yasunari Shidama. Intersections of intervals and balls in $\mathcal{E}_{\mathrm{T}}^{n}$. Formalized Mathematics, 12(3):301-306, 2004.

[13] John M. Lee. Introduction to Topological Manifolds. Springer-Verlag, New York Berlin Heidelberg, 2000.

[14] Robert Milewski. Bases of continuous lattices. Formalized Mathematics, 7(2):285-294, 1998.

[15] Beata Padlewska. Locally connected spaces. Formalized Mathematics, 2(1):93-96, 1991.

[16] Beata Padlewska and Agata Darmochwał. Topological spaces and continuous functions. Formalized Mathematics, 1(1):223-230, 1990.

[17] Karol Pąk. Basic properties of metrizable topological spaces. Formalized Mathematics, 17(3):201-205, 2009, doi: 10.2478/v10037-009-0024-8.

[18] Bartłomiej Skorulski. First-countable, sequential, and Frechet spaces. Formalized Mathematics, 7(1):81-86, 1998.

[19] Wojciech A. Trybulec. Vectors in real linear space. Formalized Mathematics, 1(2):291-296, 1990.

[20] Zinaida Trybulec. Properties of subsets. Formalized Mathematics, 1(1):67-71, 1990.

Received August 17, 2010 